\title{
Limiar anaeróbico de 4,0mM é capaz de estimar a máxima fase estável de lactato de jogadores de futebol em testes de campo
}

\author{
Anaerobic threshold of $4.0 \mathrm{mM}$ is able to estimate the maximal lactate \\ steady state of soccer players on the field tests
}

\author{
COELHO, D B; MORANDI, R F; PAIXÃO, R C; MENDES, T T; BORBA, D A; DE- \\ BARROS, C L M; PEREIRA, E R; CABIDO, C E T; GARCIA, E S. Limiar anaeróbico \\ de 4,0 mM é capaz de estimar a máxima fase estável de lactato de jogadores de futebol \\ em testes de campo. R. bras. Ci. e Mov 2015;23(2):32-39.
}

\begin{abstract}
RESUMO: Diante à importância da capacidade aeróbia para o desempenho de jogadores de futebol, o Limiar Anaeróbio tem sido um parâmetro comumente utilizado para a avaliação desses atletas. Todavia, faltam informações a respeito da validade dos protocolos de campo adotados no meio futebolístico. Portanto, o objetivo do presente estudo foi comparar os valores de velocidade de corrida (VC) e frequência cardíaca (FC) associados à máxima fase estável de lactato (MFEL) e limiar anaeróbico de 4,0mM (OBLA), e verificar se uma concentração fixa de lactato de $4 \mathrm{mM}$ pode ser utilizada para estimar a MFEL em jogadores de futebol em testes de campo. Para tanto 22 jogadores de futebol da categoria sub 20 anos $(18,1 \pm 0,2$ anos) foram avaliados. O OBLA foi considerado como a VC correspondente a lactatemia de $4,0 \mathrm{mM}$ e a MFEL foi determinada por meio de exercícios submáximos de intensidade constante com duração de 30 minutos. Os resultados não demonstraram diferenças significativas entre VC e FC identificadas pela MFEL e OBLA e apresentaram correlação significativa entre VC identificada pela MFEL e OBLA ( $r=0,56 ; p<0,01)$. Conclui-se que a concentração fixa de lactato $4 \mathrm{mM}$ (OBLA) pode ser utilizada para estimar a VC associada à MFEL em jogadores de futebol em testes de campo. A FC deve ver utilizada com cautela quanto aplicada isoladamente para controle de intensidade.
\end{abstract}

Palavras-chave: Metabolismo; Limiar de lactato; Futebol.

\begin{abstract}
Given the importance of aerobic capacity for the performance of soccer players, the Anaerobic Threshold has been a commonly parameter used to assess these athletes. However, there is missing information about the validity of field protocols adopted in the soccer environment. Therefore, the aim of this study was to compare the values of running speed (RS) and heart rate (HR) associated with the maximal lactate steady state (MLSS) and anaerobic threshold of 4.0mM (OBLA), and identify whether a fixed lactate concentration of $4 \mathrm{mM}$ can be used to estimate the MLSS in soccer players in field tests. Twenty two soccer players of under $20(18.1 \pm 0.2$ years $)$ were evaluated. OBLA was taken as the RS corresponding to blood lactate concentration of $4.0 \mathrm{mM}$ and MLSS was determined by sub maximal constant intensity exercise with a duration of 30 minutes. The results showed no significant differences between RS and HR identified by the MLSS and OBLA and showed significant correlation between the RS identified by MLSS and OBLA ( $r=0.56, \mathrm{p}<0.01)$. It is concluded that fixed concentration of $4 \mathrm{mM}$ lactate (OBLA) can be used to estimate the RS associated with MLSS in soccer players in field tests. HR should be used with caution when applied separately for intensity control.
\end{abstract}

Key Words: Metabolism; Lactate threshold; Soccer.
Daniel Barbosa Coelho ${ }^{1}$ Rodrigo Figueiredo Morandi ${ }^{2}$ Rodney Coelho da Paixão ${ }^{3}$ Thiago Teixeira Mendes ${ }^{2,4}$ Diego Alcântara Borba ${ }^{5}$ Cristiano Lino Monteiro DeBarros $^{6}$

Emerson Rodrigues Pereira ${ }^{2,7}$ Christian Emmanuel Torres Cabido $^{2}$

Emerson Silami Garcia ${ }^{4}$

${ }^{1}$ Centro Desportivo da

Universidade Federal de Ouro Preto

${ }^{2}$ Escola de Educação Física, Fisioterapia e Terapia Ocupacional da Universidade Federal de Minas Gerais ${ }^{3}$ Departamento de Ciências do Esporte, Universidade Federal do Triângulo Mineiro ${ }^{4}$ Universidade Federal do Maranhão ${ }^{5}$ Fundação Universidade de Itaúna

${ }^{6}$ Universidade Federal de Uberlândia. Instituto de Ciências Biomédicas, Faculdade de Educação Física

${ }^{7}$ Centro Universitário de Sete Lagoas, UNIFEMM

Recebido: 13/02/2014

Aceito: 19/01/2015

Contato: Christian Emmanuel Torres Cabido - christianemmanuel@gmail.com 


\section{Introdução}

O futebol é considerado uma atividade intermitente de alta intensidade na qual o desempenho pode ser determinado por componentes técnicos, táticos, psicológicos $^{1}$ e fisiológicos ${ }^{2}$. No aspecto fisiológico, embora as ações decisivas do jogo tenham característica anaeróbia (força e potência muscular), durante a partida de futebol há um predomínio do metabolismo aeróbio ${ }^{3,4}$. Desta forma, o treinamento e desenvolvimento da capacidade aeróbica deve ser planejado, em função de sua associação com o desempenho físico no futebol ${ }^{1,5}$, com a distância percorrida e o número de sprints.

Para avaliação da capacidade aeróbica, tradicionalmente o consumo máximo de oxigênio $\left(\mathrm{VO}_{2 \max }\right)$ é utilizado como parâmetro desta medida e também para explicar diferenças relacionadas ao desempenho físico entre jogadores de futebol. Entretanto, o limiar anaeróbio (Lan) parece ter maior sensibilidade para a determinação da capacidade aeróbia e desempenho físico, mesmo em situações de $\mathrm{VO}_{2 \max }$ similar ${ }^{6}$. Desta forma, a identificação de parâmetros associados ao Lan são importantes para diagnóstico e treinamento das equipes, já que a capacidade de executar exercício intermitente de alta intensidade está relacionada com a oferta de glicogênio muscular ${ }^{7}$ e com a taxa de ressíntese de ATP a partir de fosfocreatina ${ }^{8}$, sendo que a disponibilidade destes substratos energéticos parece ser influenciada pelo nível de treinamento aeróbio ${ }^{9,10}$ e intensidade de exercício associada ao $\mathrm{Lan}^{11}$.

Embora, o futebol apresente uma característica intermitente e o treinamento contínuo não seja frequentemente utilizado, a determinação do Lan tem como objetivo identificar uma velocidade de corrida ou intensidade de exercício na qual existe um desequilíbrio entre a produção e remoção de lactato ${ }^{12}$, e esta intensidade está associada com o desempenho físico em diferentes esportes que têm como base a locomoção humana ${ }^{12-14}$ e o próprio desempenho físico no futebol ${ }^{11}$.

A determinação da máxima fase estável de lactato (MFEL) é considerada o "padrão ouro" para avaliação do Lan $^{15}$. Porém a MFEL é um método dispendioso e pouco prático, visto que os sujeitos têm que comparecer ao laboratório ou sessões de testes por várias vezes, o que pode não ser compatível com o planejamento do treinamento. Vários trabalhos sugerem métodos para estimar a MFEL pela realização de apenas um exercício progressivo, seja através de respostas ventilatórias ${ }^{16}$ ou da lactatemia $^{17,18}$. Embora a utilização de protocolos de exercício com intensidade progressiva possa simplificar a estimativa da MFEL e determinação do Lan, muitos daqueles existentes na literatura ainda não foram testados em comparação com a MFEL (padrão ouro), o que, de fato, pode limitar a validade e utilização desses protocolos ${ }^{15}$.

Um método com grande aplicação prática é a utilização de concentrações fixas de lactato para determinação do Lan durante o exercício progressivo. Heck et $a l .{ }^{18}$ propuseram que a MFEL poderia ser estimada através da concentração média de lactato de 3,5 ou 4 mM (OBLA - onset of blood lactate accumulation) durante exercício de corrida. Denadai et al. $(2005)^{5}$ não encontraram diferença na velocidade de corrida em esteira identificada pelo OBLA e MFEL em jogadores de futebol em medida realizada em laboratório, o que muitas das vezes não seria compatível com a rotina de treinamento ou estrutura das equipes esportivas. No intuito de aproximar a determinação do Lan à realidade das equipes de futebol, Coelho et al. (2009) ${ }^{19}$ realizaram uma proposta de identificação do OBLA, a partir da concentração fixa de $4 \mathrm{mM}$, em testes de campo, mas não verificaram a validade da determinação da MFEL, o que poderia limitar sua utilização ${ }^{15}$. Nesse contexto, ainda não foram encontrados trabalhos na literatura que investigaram a relação entre OBLA e MFEL em testes de campo para jogadores de futebol.

Assim, o objetivo do presente estudo foi, através de um estudo descritivo e transversal, comparar os valores de velocidade de corrida (VC) e frequência cardíaca (FC) associados à MFEL e ao OBLA de jogadores de futebol em testes de campo, e verificar se uma concentração fixa de lactato de $4 \mathrm{mM}$ pode ser utilizada para estimar MFEL.

\section{Materiais e Métodos}




\section{Sujeitos}

Participaram do estudo 22 jogadores de futebol da categoria sub-20 anos vinculados a uma equipe da primeira divisão e inscrita na Confederação Brasileira de Futebol (CBF) (amostra de conveniência). Cada voluntário e, se necessário, seus pais foram informados sobre os procedimentos do experimento e suas implicações, tendo assinado um termo de consentimento livre e esclarecido para a participação no estudo. $O$ protocolo foi aprovado pelo Comitê de Ética em Pesquisa (COEP) da Universidade Federal de Minas Gerais (ETIC291/09) e respeitou todas as normas estabelecidas pelo Conselho Nacional de Saúde (Resolução 466/12) envolvendo pesquisas com seres humanos. As características gerais dos participantes são apresentadas na Tabela 1.

Tabela 1. Características gerais da amostra $(n=22)$.

\begin{tabular}{|c|c|c|c|c|c|c|}
\hline $\begin{array}{l}\text { Idade } \\
\text { (anos) }\end{array}$ & $\begin{array}{l}\text { Estatura } \\
(\mathrm{cm})\end{array}$ & $\begin{array}{l}\mathrm{MC} \\
(\mathrm{kg})\end{array}$ & $\% \mathrm{GC}$ & TT (anos) & $\begin{array}{c}\text { Treino/Semana } \\
\text { (horas) }\end{array}$ & $\begin{array}{c}\mathrm{VO}_{2 \max } \\
(\mathrm{ml} / \mathrm{kg} / \mathrm{min})\end{array}$ \\
\hline $18,1 \pm 0,2$ & $175,2 \pm 5,5$ & $69,8 \pm 4,3$ & $7,8 \pm 3,6$ & $5,5 \pm 4,0$ & 16 & $57,8 \pm 5,8$ \\
\hline
\end{tabular}

\section{Procedimentos experimentais}

Os voluntários foram submetidos a uma avaliação inicial de massa corporal, estatura, composição corporal e estimativa do $\mathrm{VO}_{2 \max }$. A massa corporal foi medida com os voluntários descalços e vestindo apenas um short, utilizando-se uma balança mecânica (Welm®) com precisão de $0,1 \mathrm{~kg}$, e a estatura foi medida em um estadiômetro acoplado a balança com precisão de $0,5 \mathrm{~cm}$. As dobras cutâneas subescapular, tricipital, peitoral, subaxilar, suprailíaca, abdominal e coxa foram medidas com um plicômetro (Lange $\AA)$, graduado em milímetros para determinação da composição $\operatorname{corporal}^{20}$. O VO $\mathrm{VO}_{2 \max }$ dos atletas foi estimado em um teste de campo, o qual consistiu em correr $2400 \mathrm{~m}$ o mais rápido possível ${ }^{21}$. A coleta de dados foi realizada durante o início da temporada de treinamento.

A determinação do OBLA foi realizada em um único dia da semana e a determinação da MFEL foi realizada na semana seguinte, em dias diferentes e intercalados por 48 horas de recuperação. Todos os testes foram realizados no mesmo local de treinamento e no período da manhã para evitar influência do ritmo circadiano. Os atletas foram orientados a não participar de atividade física intensa no dia anterior aos testes, realizaram a ingestão de $500 \mathrm{ml}$ de água na noite anterior e duas horas antes do teste para garantir o estado de hidratação, não realizar a ingestão de alimentos contendo cafeína ou outro estimulante, e se alimentarem normalmente para garantir a reposição do glicogênio muscular (dieta fornecida pela equipe/clube). Antes de cada teste, os sujeitos completaram um período preparatório de 10 minutos composto por trotes, corridas com mudança de direção, saltos, corridas em alta velocidade de curta duração e alguns exercícios de alongamento.

\section{Métodos para identificação do OBLA e MFEL}

Para identificar OBLA e MFEL foram utilizados testes de campo em terreno gramado, em uma pista de formato oval e demarcada a cada $50 \mathrm{~m}$. Todos os atletas foram familiarizados com o teste e estavam calçados com chuteiras durante a sua realização. A velocidade de corrida foi controlada a cada $50 \mathrm{~m}$ através de estímulos verbais $^{19}$, de forma que a cada marcação o atleta era orientado ajustar a velocidade (quando necessário) para que a mesma estivesse dentro do proposto para aquele atleta. Também foi registrado o tempo gasto por cada atleta em cada trecho para verificação da velocidade média ao longo do percurso e desta forma, evitar que a velocidade real de corrida, fosse diferente da planejada. Em caso de diferença maior do que $0,5 \mathrm{~km} / \mathrm{h}$ o atleta era instruído a percorrer novamente o percurso na velocidade pré determinada. 
35 Limiar anaeróbico de 4,0mM e máxima fase estável de lactato de jogadores de futebol em testes de campo

O OBLA foi considerado como a velocidade de corrida correspondente a lactatemia de $4,0 \mathrm{mM}^{18,22,23} . \mathrm{O}$ teste consistiu em duas a cinco corridas de 1000m com ritmo constante. As velocidades eram aumentadas progressivamente em $1 \mathrm{~km} / \mathrm{h}$ até ser possível a identificação da velocidade correspondente a 4,0mM de lactato através de interpolação linear ${ }^{19}$. A FC foi monitorada durante todo o teste através de um cardiofrequencímetro (Polar Electro Oy, Vantage, Finland). Entre 60 e 90 segundos após o término de cada corrida, uma amostra de sangue $(25 \mu \mathrm{L})$ era coletada da popa digital e a lactatemia analisada por meio de um lactímetro portátil (Accusport). Caso a concentração de lactato alcançasse ou superasse o valor de $4 \mathrm{mM}$, o teste era interrompido. Caso contrário, o voluntário realizava mais uma corrida com um incremento de $1 \mathrm{~km} / \mathrm{h}$ na velocidade. Para garantir que a lactatemia não ultrapassasse $4 \mathrm{mM}$ na primeira corrida, o teste iniciou a $10 \mathrm{~km} / \mathrm{h}^{19}$. Os valores de FC e concentração de lactato registrados durante $\mathrm{o}$ teste foram posteriormente analisados utilizando-se o software Microsoft ${ }^{\circledR}$ Excel 2000 para a realização da interpolação linear e determinação do OBLA.

A MFEL foi determinada por meio de exercícios submáximos de intensidade constante com duração de 30 minutos. A MFEL foi considerada como a mais alta concentração sanguínea de lactato e velocidade de corrida na qual a lactatemia não apresentou aumento superior a $1 \mathrm{mM}$ durante os vinte minutos finais de exercício ${ }^{18,24-26}$. A primeira intensidade escolhida foi àquela correspondente ao OBLA identificado previamente. Se durante o primeiro teste um estado estável ou uma diminuição da lactatemia fosse observada, a intensidade dos testes subsequentes era aumentada até que o estado estável do lactato não pudesse mais ser observado. Caso a lactatemia durante a realização do primeiro teste não apresentasse um estado estável e/ou ocorresse a fadiga do voluntário antes do término do teste, as intensidades subsequentes eram reduzidas. A precisão adotada foi de 1 $\mathrm{km} / \mathrm{h}$. Amostras de sangue $(25 \mu \mathrm{L})$ foram coletadas da popa digital em repouso e nos minutos 10 e 30 de exercício para determinação da lactatemia. Para FC e lactatemia correspondentes à MFEL foram consideradas as médias dos minutos 10 e 30 de exercício.

\section{Análise estatística}

Inicialmente foram verificadas a normalidade de distribuição dos dados e homocedasticidade através dos testes de Shapiro-Wilk e Levene. Para comparação da VC e FC identificadas pela MFEL e OBLA foi utilizado o teste $\mathrm{t}$ de student pareado ${ }^{5}$. Para testar a associação entre os resultados encontrados, foi utlizada a correlação do produto momento de Pearson. Foi adotado nível de significância de $\alpha<0,05$. Todos os resultados estão apresentados como média \pm desvio padrão.

\section{Resultados}

Não foram encontradas diferenças significativas para VC e FC identificadas a partir da MFEL e do OBLA $(\mathrm{p}=0,14$ e $\mathrm{p}=0,21$, respectivamente) (Tabela 2$)$.

Tabela 2. Velocidade de corrida (VC) e frequência cardíaca (FC) identificadas na máxima fase estável de lactato (MFEL) e onset of blood lactate accumulation (OBLA).

\begin{tabular}{|c|c|c|c|}
\hline & VC $\left(\mathrm{km} \cdot \mathrm{h}^{-1}\right)$ & FC (bpI & \\
\hline MFEL & $11,8 \pm 1,1$ & 179 & 8 \\
\hline OBLA & $12,3 \pm 1,6$ & $175 \pm$ & 14 \\
\hline
\end{tabular}

Foi observada correlação significativa entre as VC identificadas pela MFEL e OBLA $(r=0,56 ; \mathrm{p}<0,01)$ (Figura 1A). O mesmo não ocorreu para os valores de FC $(\mathrm{r}=0,04 ; \mathrm{p}>0,05)$ (Figura 1B).

O valor médio de lactato encontrado na MFEL (5,0 $\pm 2,4 \mathrm{mM}$ ) no presente estudo foi maior do que o utilizado a concentração fixa utilizada para identificar o OBLA (4,0mM). A lactatemia na MFEL apresentou variação individual de 1,3 a $9,3 \mathrm{mM}$. 

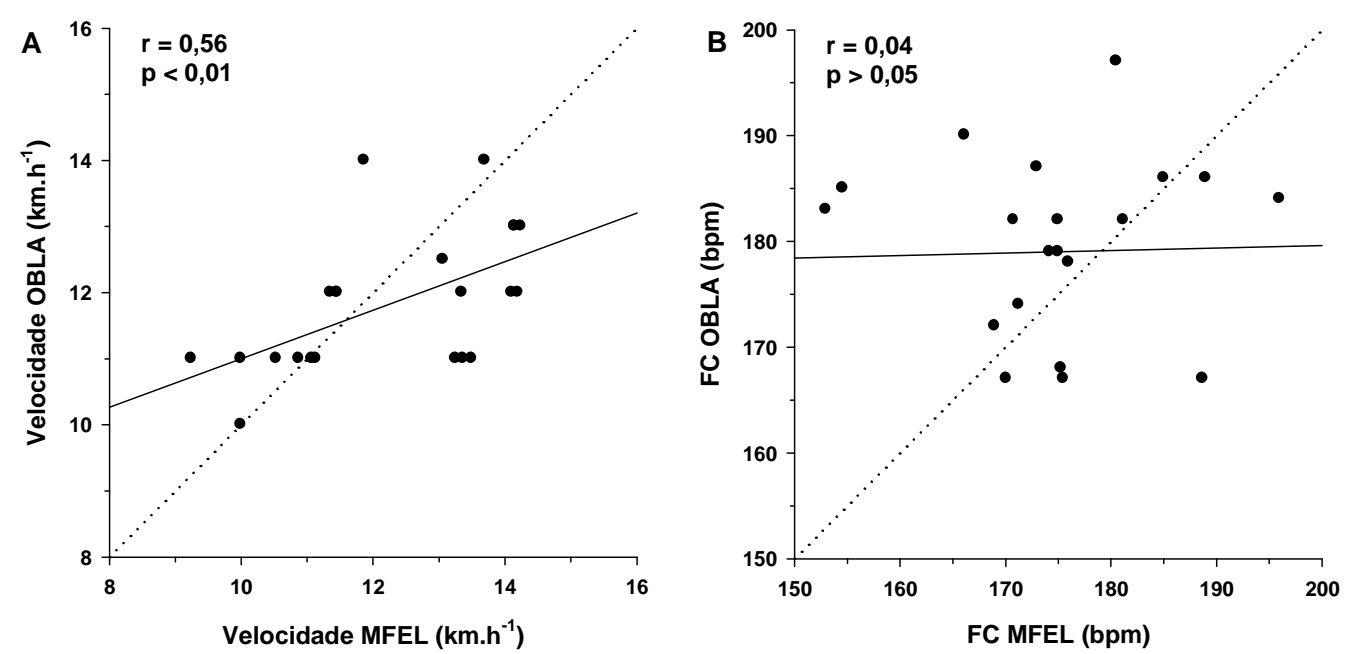

Figura 1. Correlação entre velocidade de corrida (A) e frequência cardíaca (B) identificada pela máxima fase estável de lactato (MFEL) e onset of blood lactate accumulation (OBLA).

\section{Discussão}

O principal achado do presente estudo é que o OBLA foi capaz de estimar a VC associada a MFEL de jogadores de futebol da categoria sub-20 durante testes de campo. Denadai et $a l^{5}$ também não encontraram diferença na VC determinada pelo OBLA e MFEL durante testes em esteira em jogadores de futebol. Outros trabalhos também não encontraram diferença entre a intensidade de exercício identificada pelo OBLA e MFEL $^{26-27}$. Entretanto, Foxdal et al. ${ }^{28}$ constataram que o OBLA superestimou a MFEL em corredores de resistência altamente treinados e Urhausen et al. $^{29}$ demonstraram que apenas $56 \%$ dos voluntários, estavam realmente na intensidade da MFEL quando se exercitavam na intensidade do OBLA. Especificamente com jogadores de futebol, McMillan et al. ${ }^{30}$ e Silva et $a l .{ }^{31}$ também avaliaram o OBLA em jogadores, por meio de testes laboratoriais, mas sem comparação direta com a MFEL.

A VC encontrada no presente estudo $(12,3 \pm 1,6$ $\mathrm{km} / \mathrm{h}$ ) foi menor daquelas encontradas em outros trabalhos que determinaram o OBLA em jogadores de futebol na pré temporada e período competitivo de jogadores profissionais $(13,62 \pm 0,25$ e $14,67 \pm 0,24$ $\mathrm{km} / \mathrm{h})^{30}$, em jogadores da série A3 $(14,28 \pm 1,02 \mathrm{~km} / \mathrm{h})^{31} \mathrm{e}$, em jogadores profissionais $(13,6 \pm 1,4 \mathrm{~km} / \mathrm{h})^{5}$. Entretanto, as diferenças encontradas entre as diferentes amostras podem estar relacionadas ao regime de treinamento das equipes em função da amostra/categoria estudada, em função das maiores exigências físicas da categoria profissional.

De fato, a partir dos resultados encontrados no trabalho atual, parece que o OBLA é capaz de estimar a MFEL em jogadores de futebol, reforçando a possibilidade de utilizar esta metodologia em testes de campo, em condições próximas à realizada na prática e fora de condições laboratoriais, como anteriormente proposto por Coelho et al. ${ }^{19}$, como também em condições laboratoriais, como proposto por Denadai et al. ${ }^{5}$. Desta forma, esta metodologia permite a incorporação de avaliações do Lan dentro de um contexto mais próximo da realidade do futebol, o que pode permitir a maior utilização desta medida pelos clubes, como forma de diagnóstico de sua equipe quanto à condição física, como também definição de intensidade de exercício para o treinamento físico.

Também não foram encontradas diferenças significativas para a FC na MFEL e OBLA, embora não tenha sido encontrada correlação significativa entre os dois protocolos (Figura 1B). Deste modo, deve-se ter cautela ao utilizar a FC determinada pelo OBLA como variável de controle ou prescrição do treinamento, já que em alguns casos a FC pode diferir daquela encontrada. Embora na prática os treinamentos sejam frequentemente 
37 Limiar anaeróbico de 4,0mM e máxima fase estável de lactato de jogadores de futebol em testes de campo

controlados pela resposta da FC, em função da facilidade de medida e elevado número de equipamentos disponíveis, devemos ter cautela na utilização desta variável quando associada ao Lan, bem como em função de diferentes fatores que podem influenciar a resposta da FC, como a temperatura ambiente, hidratação, variação diária $^{32}$. A não correlação significativa entre FC no OBLA e MFEL talvez possa ser explicada pela configuração do teste de campo, o qual teve uma distância pré determinada, o que causa modificação do tempo de corrida para cada velocidade. Outros estudos deverão investigar a possibilidade de corrida por um tempo constante, em detrimento a uma determinada distância, na determinação da VC e FC pelo OBLA.

A possibilidade de utilização da FC para controle ou prescrição de treinamento é uma importante ferramenta em detrimento às dificuldades de controle da VC, embora o número de equipamentos para o controle desta variável esteja cada vez mais presentes em nossa realidade em função da difusão dos dispositivos com GPS (global positioning system - sistema de posicionamento global), embora as possíveis análises sejam menos frequentes e mais complexas. Em relação ao método utilizado no presente estudo para controle de VC, para realização do teste para determinação do Lan, o controle verbal embora apresente grande praticidade, seja utilizado no dia a dia e não necessidade de maiores investimentos em equipamentos, o mesmo pode apresentar uma variação em relação a velocidade pré determinada e a que o atleta está realmente desenvolvendo. Para minimizar estes problemas, é necessário que seja registrado o tempo total de corrida e o tempo em cada trecho do percurso para verificar a velocidade média de corrida e, se necessário, repetir a situação. No presente trabalho, não foi realizado nenhum tipo de controle relacionado a VC e sugerimos que futuros trabalhos utilizem de maneira concomitante o GPS para controle de VC.

É importante ressaltar que a lactatemia identificada na MFEL $(5,0 \pm 0,5 \mathrm{mM})$ foi maior do que aquela utilizada para identificar o OBLA $(4,0 \mathrm{mM})$. Tal fato está de acordo com o resultados observados por Beneke ${ }^{24}$
$(5,1 \mathrm{mM})$ que também encontrou valor superior a $4,0 \mathrm{mM}$ na MFEL. Isso pode ser explicado pelo reduzido tempo de corrida em cada velocidade (4-6 min) o que não permite um completo equilíbrio entre a produção e difusão do lactato para a corrente sanguínea ${ }^{28}$, e desta forma uma concentração de lactato menor durante o teste para determinação do OBLA em relação aos testes constantes para determinação da MFEL. A variação de lactatemia observada para MFEL no presente estudo (1,3 a 9,3mM) reforça a grande variabilidade individual desta medida, conforme já demonstrado na literatura ${ }^{18,26,27,33}$.

Considerando a importância em se avaliar a capacidade física dos atletas de futebol e a relevância da medida do Lan, a utilização do OBLA em testes de campo permite que o Lan seja determinado em condições mais próximas e compatíveis com a rotina de treinamento das equipes. Entretanto, as informações apresentadas acima estão limitadas à comparação entre MFEL e OBLA para um momento específico da temporada. Bem como às características do teste utilizado, corrida contínua. Pesquisas futuras devem investigar se o OBLA é capaz de avaliar as adaptações do treinamento e se mantém a associação com a MFEL em situações de médio e longo prazo.

A extrapolação dos resultados deste estudo precisam considerar as limitações presentes no mesmo, destacando o número de voluntários estudados. Por se tratar de um clube de futebol profissional, o número de voluntários dependia de quantidade de atletas que estavam treinando regularmente no clube no período em que o estudo foi realizado, nesse caso 22 atletas. Assim, estudos futuros deveriam utilizar um maior número de voluntários para que tais resultados sejam mais conclusivos.

\section{Conclusões}

O OBLA (4,0mM) foi capaz de estimar a MFEL de jogadores de futebol em testes de campo de $1.000 \mathrm{~m}$ e pode ser utilizado como um método para avaliação da condição física dos atletas como também para a definição de zonas de intensidade de exercício. 


\section{Referências}

1. Corrêa DKDA, Alchieri JC, Duarte LRS, Strey MN. Excelência na produtividade: a performance dos jogadores de futebol profissional. Psicologia: Reflexão e Crítica. 2002;15:447-60.

2. Hoff J, Wisloff U, Engen LC, Kemi OJ, Helgerud J. Soccer specific aerobic endurance training. Br J Sports Med. 2002;36(3):218-21.

3. Bangsbo J. Energy demands in competitive soccer. J Sports Sci. 1994;12 Spec No:S5-12.

4. Bangsbo J, Norregaard L, Thorso F. Activity profile of competition soccer. Can J Sport Sci. 1991;16(2):110-6.

5. Denadai BS, Gomide EB, Greco CC. The relationship between onset of blood lactate accumulation, critical velocity, and maximal lactate steady state in soccer players. J Strength Cond Res. 2005;19(2):364-8.

6. Ziogas GG, Patras KN, Stergiou N, Georgoulis AD. Velocity at lactate threshold and running economy must also be considered along with maximal oxygen uptake when testing elite soccer players during preseason. J Strength Cond Res. 2011;25(2):414-9.

7. Gaitanos GC, Williams C, Boobis LH, Brooks S. Human muscle metabolism during intermittent maximal exercise. J Appl Physiol. 1993;75(2):712-9.

8. Glaister M, Lockey RA, Abraham CS, Staerck A, Goodwin JE, McInnes G. Creatine supplementation and multiple sprint running performance. J Strength Cond Res. 2006;20(2):273-7.

9. Constable SH, Favier RJ, McLane JA, Fell RD, Chen M, Holloszy JO. Energy metabolism in contracting rat skeletal muscle: adaptation to exercise training. Am J Physiol. 1987;253(2 Pt 1):C316-22.

10. Yoshida T. The rate of phosphocreatine hydrolysis and resynthesis in exercising muscle in humans using $31 \mathrm{P}-$ MRS. J Physiol Anthropol Appl Human Sci. 2002;21(5):247-55.

11. Helgerud J, Engen LC, Wisloff U, Hoff J. Aerobic endurance training improves soccer performance. Med Sci Sports Exerc. 2001:33(11):1925-1931.

12. Billat VL. Use of blood lactate measurements for prediction of exercise performance and for control of training: Recommendations for long-distance running. Sports Med. 1996:22(3):157-75.

13. Beneke R. Anaerobic threshold, individual anaerobic threshold, and maximal lactate steady state in rowing. Med Sci Sports Exerc. 1995:27(6):863-7.

14. Beneke R, Hütler M, Leithäuser RM. Maximal lactate-steady-state independent of performance. Med Sci Sports Exerc. 2000:32(6):1135-1139.

15. Faude O, Kindermann W, Meyer T. Lactate threshold concepts: how valid are they? Sports Med. 2009;39(6):46990.

16. Wasserman K, McIlroy MB. Detecting the Threshold of Anaerobic Metabolism in Cardiac Patients during Exercise. The American J of Cardiol. 1964;14:844-52.

17. Stegmann H, Kindermann W, Schnabel A. Lactate kinetics and individual anaerobic threshold. Int J Sports Med. 1981;2(3):160-5.

18. Heck H, Mader A, Hess G, Mucke S, Muller R, Hollmann W. Justification of the 4-mmol/1 lactate threshold. Int J Sports Med. 1985;6(3):117-30.

19. Coelho DB, Mortimer LÁ, Condessa LA, Soares DD, De Barros CLM, Garcia ES. Limiar anaeróbio de jogadores de futebol de diferentes categorias. Rev Bras Cineantropom Desempenho Hum. 2009;11(1):81-7.

20. Jackson AS, Pollock ML. Generalized equations for predicting body density of men. Br J Nutr. 1978;40(3):497504.

21. Margaria R, Aghemo P, Pinera Limas F. A simple relation between performance in running and maximal aerobic power. J Appl Physiol. 1975;38(2):351-2.

22. McMillan K, Helgerud J, Grant SJ, Newell J, Wilson J, Macdonald R, et al. Lactate threshold responses to a season of professional British youth soccer. Br J Sports Med. 2005;39(7):432-6.

23. Silva ASRD, Santos FNCD, Santhiago V, Gobatto CA. Comparação entre métodos invasivos e não invasivo de determinação da capacidade aeróbia em futebolistas profissionais. Rev Bras de Med do Esporte. 2005;11:233-7.

24. Beneke R. Methodological aspects of maximal lactate steady state-implications for performance testing. Eur $\mathbf{J}$ Appl Physiol. 2003;89(1):95-9.

25. Billat VL, Sirvent P, Py G, Koralsztein JP, Mercier J. The concept of maximal lactate steady state: a bridge between biochemistry, physiology and sport science. Sports Med. 2003;33(6):407-26. 
39 Limiar anaeróbico de 4,0mM e máxima fase estável de lactato de jogadores de futebol em testes de campo

26. Denadai BS, Figueira TR, Favaro OR, Goncalves M. Effect of the aerobic capacity on the validity of the anaerobic threshold for determination of the maximal lactate steady state in cycling. Braz J Med Biol Res. 2004;37(10):1551-6.

27. Van Schuylenbergh R, Vanden Eynde B, Hespel P. Correlations between lactate and ventilatory thresholds and the maximal lactate steady state in elite cyclists. Int J Sports Med. 2004;25(6):403-8.

28. Foxdal P, Sjodin A, Sjodin B. Comparison of blood lactate concentrations obtained during incremental and constant intensity exercise. Int J Sports Med. 1996;17(5):360-5.

29. Urhausen A, Coen B, Weiler B, Kindermann W. Individual anaerobic threshold and maximum lactate steady state. Int J Sports Med. 1993;14(3):134-9.

30. McMillan K, Helgerud J, Grant SJ, Newell J, Wilson J, Macdonald R, et al. Lactate threshold responses to a season of professional British youth soccer. Br J Sports Med. 2005;39(7):432-6.

31. Silva ASRD, Santos FNCD, Santhiago V, Gobatto CA. Comparação entre métodos invasivos e não invasivo de determinação da capacidade aeróbia em futebolistas profissionais. Rev Bras de Med do Esporte. 2005;11:233-7.

32. Achten J, Jeukendrup AE. Heart Rate Monitoring. Applications and Limitations. Sports Med. 2003:33(7):517-538.

33. Baron B, Dekerle J, Robin S, Neviere R, Dupont L, Matran R, et al. Maximal lactate steady state does not correspond to a complete physiological steady state. Int J Sports Med. 2003;24(8):582-7. 